\title{
Sleep Board Review Question: Hyperarousal in Insomnia
}

\section{Rohit Budhiraja, MD}

Department of Medicine, Southern Arizona Veterans Affairs Health Care System (SAVAHCS) and University of Arizona, Tucson, AZ.

Insomnia is characterized by which of the following?

1. Increased brain metabolism compared to controls only during awake.

2. Increased brain metabolism compared to controls only during sleep.

3. Increased brain metabolism during both asleep and awake compared to controls.

4. No increase in brain metabolism compared to controls. 


\section{Correct!}

\section{Increased brain metabolism during both asleep and awake compared to controls.}

Insomnia is a condition of physiological hyperarousal during both sleep and wakefulness $(1,2)$. There is increased activation of somatic, cortical and cognitive functioning during the day as well as night.

- Insomniacs have elevated heart rate compared to good sleepers across the sleep-wake cycle

- Body temperature and blood pressure are higher in insomniacs.

- There is a nocturnal and diurnal increase in the whole-body metabolic rate.

- Levels of cortisol and adrenocorticotropic hormone during sleep are elevated.

- Insomniacs show increased high-frequency electroencephalographic (EEG) beta and sleep spindle (sigma) activity during non-rapid eye movement sleep.

- Alpha and beta EEG frequencies are increased both in wake and sleep.

- One study utilized [(18)F]Fluorodeoxyglucose positron emission tomography to assess regional cerebral glucose metabolism in 7 patients with insomnia and 20 healthy subjects (3). Compared with healthy subjects, patients with insomnia showed greater global cerebral glucose metabolism during sleep and while awake. There was also a smaller decline in relative metabolism from waking to sleep states in wake-promoting regions compared to controls.

\section{References}

1. Buysse DJ. Insomnia. JAMA. 2013;309:706-16. [CrossRef] [PubMed]

2. Bonnet MH, Arand DL. Hyperarousal and insomnia: state of the science. Sleep Med Rev. 2010;14:9-15. [CrossRef] [PubMed]

3. Nofzinger EA, Buysse DJ, Germain A, Price JC, Miewald JM, Kupfer DJ. Functional neuroimaging evidence for hyperarousal in insomnia. Am J Psychiatry. 2004;161:2126-8. [CrossRef] [PubMed] 